\title{
¿Destrabando una sostenibilidad estéril? Algunas consideraciones en el proceso de selección de indicadores para un diagnóstico territorial
}

\section{Felipe Gutiérrez-Antinopai}

Licenciado en Construcción Civil por la Universidad Técnica Federico Santa María, Valparaíso Chile, y M. Sc. en Asentamientos Humanos y Medio Ambiente por la Pontificia Universidad Católica de Chile. Profesor adjunto del curso "Optativo de profundización: Sistemas de Indicadores para el Desarrollo Sustentable" en el Magíster en Desarrollo Sustentable de Ambientes y Territorios de la Universidad Academia de Humanismo Cristiano. Asociado al Núcleo de Investigación y Docencia en Ambiente y Sociedad, NIDAS. Trabajó como investigador para la fundación NEWENKO. Adicionalmente es ingeniero de Sostenibilidad en el proyecto "Plataforma de Apoyo a la Gestión de Residuos y Puntos Limpios” en la comuna de San Antonio, Chile. figutierrez@uc.cl

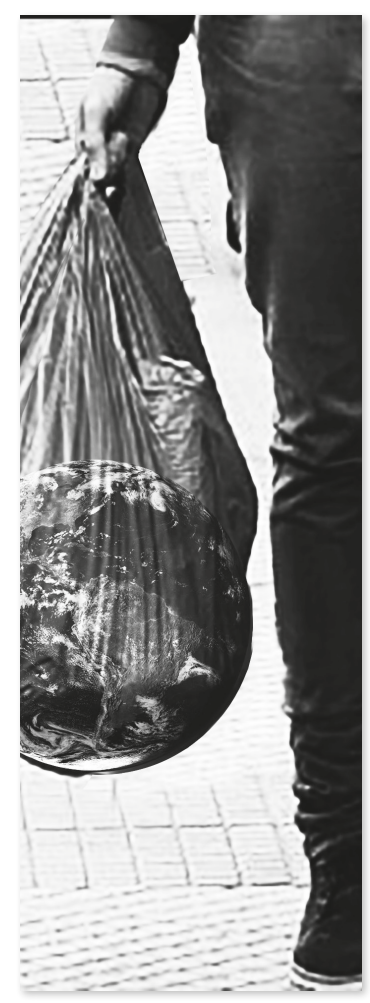




\section{¿Destrabando una sostenibilidad estéril? Algunas consideraciones en el proceso de selección de indicadores para un diagnóstico territorial}

\section{Resumen}

Cuando la sostenibilidad surgió como el consenso por antonomasia para modificar agendas gubernamentales, se levantaron variadas voces que incentivaban el uso de nuevas métricas para el diagnóstico de territorios. No obstante, gran parte de ellos promovidos por entidades gubernamentales parecen muchas veces continuar con operacionalizar la definición de sostenibilidad con enfoques que se concentran en superar aspectos materiales, lo que ha significado una lucha incesante únicamente "contra la pobreza”. De otra forma, si en la sostenibilidad subyace únicamente la idea de combatir la pobreza, ¿cómo podríamos diferenciarla de aquella que necesita restringir el consumo doméstico e industrial? Este artículo emprende una discusión en torno de los sistemas de indicadores y cómo ellos son fundamentales para traducir la sostenibilidad en un diagnóstico territorial que devele un "espacio ambiental", desafiando a replantearse prioridades que promuevan la toma de decisiones óptimas a diferentes escalas.

\section{Palabras clave}

Indicadores; espacio ambiental; sostenibilidad; diagnósticos territoriales.

\section{Unlocking a sterile sustainability? Some considerations in the process of selecting indicators for a territorial diagnosis}

\begin{abstract}
When sustainability emerged as the consensus by antonomasia to modify governmental agendas, several voices that encouraged the use of new metrics for the territorial diagnostic, were raised. However, many of them, promoted by governmental entities, often seems to continue with the operationalization of the definition of sustainability with focus on the overcome of material aspects, which has meant an incessant struggle only "against to the poverty". Otherwise, if sustainability underlies only the idea of combating poverty, how can we differentiate it from the one that needs to restrict domestic and industrial consumption? This article undertakes a discussion around system of indicators and how they are fundamental to translate sustainability into a territorial diagnosis that reveals an "environmental space", challenging to rethink priorities that promote optimal decision making at different scales.
\end{abstract}

\section{Keywords}

Indicators; environmental space; sustainability; territorial diagnosis.

\section{Desbloquear uma sustentabilidade estéril? Algumas considerações no processo de seleção de indicadores para um diagnóstico territorial}

\section{Resumo}

Quando a sustentabilidade surgiu como o consenso por antonomásia para modificar agendas governamentais, se levantaram muitas vozes que incentivavam o uso de novas métricas para o diagnóstico de territórios. Não obstante, grande parte de eles, promovidos por entidades governamentais parecem, muitas vezes, continuar com o fato de fazer operacional a definição de sustentabilidade com abordagens que se concentram na superação de aspectos materiais, o que tem significado uma luta incessante unicamente "contra a pobreza". De outro modo, si na sustentabilidade está subjacente unicamente a ideia de combater a pobreza ¿como poderíamos diferenciá-la de aquela que necessita restringir o consumo doméstico e industrial? Este artigo inicia uma discussão em torno dos sistemas e indicadores e como estes são fundamentais para traduzir a sustentabilidade num diagnóstico territorial que revele um "espaço ambiental", desafiando a replantar-se prioridades que promovam a tomada de decisões ótimas em diferentes escalas.

\section{Palavras chave}

Indicadores; espaço ambiental; sustentabilidade; diagnósticos territoriais. 


\section{¿Destrabando una sostenibilidad estéril? Algunas consideraciones en el proceso de selección de indicadores para un diagnóstico territorial}

\section{Introducción ${ }^{1}$}

Los sistemas de indicadores como herramientas metodológicas técnicas-políticas en estados de arte de desempeño o simplemente de diagnóstico territorial se pueden remontar a Alfredo Niceforo desde la criminología, y en su adopción y difusión, a la elaboración de estadísticas con Raymond Bauer y Richard Stone durante los años 30 a los 60 (Noll, 2016; GutiérReZ-Antinopai y Escalona, 2018). Algunos documentos relevantes en la adopción global de indicadores fueron Nivel de Vida, por la Organización de Naciones Unidas en el año 1954, y Hacia un Sistema de Estadísticas Sociales y Demográficos, publicado en el año 1975. Estos, ideándose en un contexto de posguerra y con el ánimo de empujar a una Europa devastada y a países en pleno ascenso económico, contribuyeron a una línea base metodológica que hoy en día aún es posible concebir en diferentes experiencias de selección de indicadores de índole social (NoLL, 2002).

Desde los 90 con la llegada del concepto sostenibilidad ${ }^{2}$ y por su rol estratégico que permitiría oficializar la inclusión de la dimensión ambiental como parte ineludible de un diagnóstico territorial, los sistemas de indicadores fueron ampliamente reconocidos como una representación del cómo hacer territorio (MEAdows, 1998; BosseL, 1999). De hecho, en Latinoamérica y el Caribe desde 1992 el 80 \% de los países echó a andar una institucionalidad ambiental, lo que significó un abre telón para la incorporación de sistemas de evaluación ambiental (GUTIÉRREZ-AnTinopaI, 2017).

Sin embargo, a más de treinta años de publicado el informe Brundtland en Latinoamérica, podemos ver una sobreutilización de sostenibilidad que ha levantado una especie de entorno polisémico que disfraza la urgencia histórica de carácter ambiental en una de ficción, localizando los indicadores como meras herramientas contextuales sin poder todavía ate$\operatorname{rrizar}^{3}$ y facilitar uno de los principales tratados: límites o finitud de recursos naturales.

En esta línea, el objetivo principal de este ensayo es retomar la importancia de los indicadores en la operacionalización de la sostenibilidad. Para ello, el artículo se divide en cuatro secciones. Primero, se manifiestan ciertas tendencias que detienen algunos fundamentos de la sostenibilidad; luego, en torno de una discusión en el quehacer de los sistemas de indicadores se conceptualiza la sostenibilidad. Tercero, mediante la elección de un marco metodológico se otorga una revisión en el uso de indicadores. Por último, a partir de la
1. Quisiera agradecer $a$ Jonathan Barton por la oportunidad de incorporarme al mundo de los indicadores en el año 2015, y además a Raúl González-Meyer, quien, por sus comentarios en la primera etapa de trabajo, sentó la estructura de este ensayo.

2. Se tomará sostenibilidad como definición central, reconociendo, sin embargo, que "sustentabilidad" es también una representación que autores en espacios Iberoamericanos han promovido, a pesar de que posiciones conceptuales fueron muchas veces obviadas. Esto se debe a dos razones: primero, por el hecho de que sostenibilidad es, actualmente, el concepto avalado por organismos intergubernamentales $y$, segundo, porque "sustentabilidad" no se incluye en la Real Academia Española.

3. De GUIMARAEs (1998), quien rescata la metáfora de BoISIER (1997). 
discusión de un caso de Chile, se intenta visibilizar la problemática funcional en el manejo institucional del uso de los sistemas de indicadores.

\section{¿Una sostenibilidad estéril?}

Sería ingenuo pensar que todos estaríamos de acuerdo en un concepto que cuenta con claros matices. El primero de ellos es haber separado las visiones dentro de lo que TURNER (1993) clasificó como sostenibilidad muy débil, débil, fuerte y muy fuerte. Así también, variados autores que promovieron el término acusaron falta de probidad, por ejemplo, en establecer límites o umbrales de consumo principalmente en los países de altos ingresos y, además, en lo que ha sido etiquetado como "economía verde", ampliamente criticada por el énfasis en el crecimiento económico (crematístico), la planificación estratégica neoliberal y el incentivo al consumo (véanse Guimaraes, 1994; Martínez-Alier, 1999; Naredo, 2007 y Lander, 2011).

Visiblemente, este escenario pareciera entregar más obstáculos que facilidades para la operacionalización del desarrollo sostenible. Las líneas de acción consensuadas en los 70 con el Informe Founex (CLub DE Roma, 1971), Una Sola Tierra: El Cuidado y Conservación de un Pequeño Planeta (WARD y DuBos, 1972) y World Conservation Strategy (IUCN, UNEP y WWF, 1980) no han servido de fundamento, y todo lo que se ha pronunciado desde entonces es un relato gatopardista que necesita continuamente nuevos conceptos, nuevos principios y nuevas acciones, de tal manera que se continúen validando indicadores que reflejen el sistema económico neoliberal-capitalista.

Uno de los indicadores, el PIB, portavoz por excelencia del enfoque neoliberal, ayuda a discernir entre aquellas economías solventes y las que no lo son. En efecto, dentro de la región latinoamericana y mundial, Chile ha llamado la atención por su crecimiento económico y principalmente por las razones e ideologías detrás de estos resultados. Milton Friedman, artífice de este modelo, califica a Chile como "el milagro económico chileno". Por medio de la liberalización de mercados y privatización de empresas logró enfrentar la pobreza, digamos, de manera exitosa a nivel comunicacional. Efectivamente, esta lucha ha sido hidalgamente llevada a cabo por diferentes coaliciones políticas, las cuales, haciendo uso público de indicadores básicos de pobreza, vivienda, cobertura de transporte y educación e incluso del Índice de Desarrollo Humano (IDH) validaron una y otra vez el sistema económico, y como resultado, se reconoce el vínculo-casi-indisoluble entre PIB-IDH y PIB-calidad de vida. 


\section{¿Destrabando una sostenibilidad estéril? Algunas consideraciones en el proceso de selección de indicadores para un diagnóstico territorial}

No obstante, pese a que la pobreza extrema y la indigencia disminuyeron y simultáneamente aumentó la longevidad promedio del país, este escenario solo se ocupa de una facción del desarrollo ${ }^{4}$. En efecto, la situación medioambiental en Chile en términos de impactos biofísicos se contrapone a la visión crematística antes mencionada. Algunos indicadores, como el Environmental Perfomance Index 2016, ubican a Chile en el número 152 de 180 países en tendencias de aumento de $\mathrm{CO} 2,75$ en el uso eficiente de nitrógeno o eficiencia en la fertilización y usos de tierras y 125 en vitalidad del ecosistema (Yale University, Columbia University y World Econonomic Forum, 2018). Otro indicador, el Happy Planet Index (New Economic FoundaTION, 2016), lo sitúa 102 de 144 países en la huella ecológica con 4,4 hectáreas globales, muy por sobre la biocapacidad que permite hasta las 1,7 hectáreas globales per cápita. Esto nos lleva a plantear que bajo el consenso del desarrollo sostenible se incrementaron los impactos ambientales, lo que trajo como consecuencia que se releguen los fundamentos de límites biofísicos y se limite visibilizar diagnósticos territoriales desde su dimensión ambiental, y desgraciadamente, se trabe el aterrizaje de la sostenibilidad.

\section{Destrabando la sostenibilidad}

Las opciones que se presentan para enfrentar este problema no solo se podrían remitir a un campo teórico, sino a uno práctico-comunicacional, entendiéndose como aquel plano donde se ejecuta y aterriza una teoría o conceptualización, que según Guimaraes (1998) sería el de los indicadores. Tal como se anunció y consensuó desde la Declaración de Estocolmo (ONU, 1972), el Informe Brundtland (WCED, 1987) y la Agenda 21 (ONU, 1992), y las actuales Metas del Desarrollo Sostenible (ONU, 2015) y Nueva Agenda Urbana (ONU, 2017), el constante monitoreo por medio del sistema de indicadores es un camino insoslayable en el proceso de desarrollo de políticas públicas en pos de las próximas generaciones. De otra manera, la utilización de indicadores permitiría conocer niveles o grados de sostenibilidad en cada ámbito, y, por tanto, apuntar a las inconsistencias de las actuales políticas públicas y ayudar a la toma de decisiones por parte de la comunidad e instituciones en general (BARTON, 2006).

El interrogante acerca de cómo construir un sistema de indicadores es el primer lente de aproximación que puede aterrizar la definición del desarrollo sostenible al cómo o qué se está midiendo. No obstante, no hay una sistematización, metodología o marco conceptual únicos a los cuales acudir para generarlo(s). De hecho, muchos de ellos no cuentan siquiera
4. Sucesivos trabajos dan cuenta de que en relación con los aspectos socioeconómicos en Chile se gestó una línea de superación en aspectos materiales, pero con cierta vulnerabilidad a las crisis económicas en regiones commodities debido al desarrollo mono-productor (véase DAHER, 2015), al aumento de desigualdades en la distribución de poder, de relacionales sociales, de segregación territorial $y$ acceso a oportunidades (véanse LARRAÑAGA Y RODRÍGUEZ, 2014; RASSE, 2017). En definitiva, las relaciones central-periférico y urbano-rural siguen siendo antagonistas en cuanto a la apropiación de un desarrollo que se plasma constantemente bajo un uso permanente de medias aritméticas en varios estudios de corte económico ortodoxo. 
5. A fines de los 60, desde la economía ecológica, ecología profunda y varios manifiestos daban importancia a la cuestión "escala". GEORGESCU ROEGEN (1996) fija su atención en las incongruencias alfanuméricas en los procesos económicos que simplifican la identificación y el comportamiento de variables en el territorio, mediante la cuantificación

y homogeneización de estadísticas. ARNE NAESSE (2007) y $D_{A L Y}$ Y COBB (1997), en la misma línea, indican que dicha incongruencia se podría resolver encontrando una escala óptima de decisiones desde lo local, cuya importancia radica en que sus actores tendrían mayores grados de control y, en consecuencia, asimilación del territorio. De ahí una de las razones por las que comunidades y hacedores de decisiones en escalas municipales sean actores preponderantes. con la definición de sistema de indicadores o indicador, e incluso con la conceptualización de lo que comúnmente se entiende como la tríada de la sostenibilidad: dimensión social, medioambiental y económica (GuTIÉRREZ-AnTINopAI, 2017), a lo cual se pueden también sumar aspectos de gobernanza.

Empecemos con lo básico. Un sistema de indicadores es una sistematización lógica desde la selección de indicadores a la comunicación, donde cada uno de ellos refleja un atributo o característica de la variable que se quiere medir (Meadows, 1998; Bossel, 1999; Scнuschnу у Sото, 2009). Segundo, dicho sistema de indicadores responderá, por lo general, a tres puntos esenciales: contexto, escala y variables. Del contexto nace la dialéctica entre el investigador y el fenómeno en cuestión, en la cual se relevan preguntas que llevan al para qué se está midiendo y cómo se comunicará cierto fenómeno (GuTiÉRREz-ANTiNOPAI, 2017). En este caso se podría avizorar si la métrica será contrastada con otros territorios. De la escala, se acota el campo de estudio; así, desde una visión de políticas públicas o socioecológica, podría remitirse a divisiones político-administrativas: comuna, región localidad, nación. Asimismo, podría realizarse desde visiones biofísicas o de paisaje si se quiere saber el estado de cuencas o grupos de especies en peligro de extinción. De esta última, la clasificación escala político-administrativa quedaría supeditada a la extensión de especies o ecosistemas en estudio. Acotar el campo de estudio tiene una clara connotación de controlar y guiar la toma de decisiones a partir de un gobierno central o hacedores de decisión ${ }^{5}$. Tercero, se identifican variables y actores principales que inciden a nivel territorial. Para ilustrar mejor, el deterioro de la salud en una zona netamente industrial podría decantar en la siguiente pregunta: ¿cuál es el impacto de la industria en la calidad de vida? Ello llevaría a conocer el telos o diagnóstico último que se experimentaría. En segundo lugar, la escala podría definirse por la cantidad de habitantes urbanos, y, por último, se requeriría saber cuáles son las relaciones entre variables y actores que estén impactando la calidad de vida para luego con la información adecuada seleccionar los indicadores y sus respectivos contrastes territoriales o su relación óptimo-real, que respondería a la pregunta ¿qué distancia hay entre lo medido con lo ideal?

Hasta aquí no debiese haber una brecha notable, según cuán diferentes sean los lentes con que se esté mirando dicha metodología. Desde la economía ortodoxa a ecológica podrían encontrarse con ella; sin embargo, la diferencia entre uno y otro difiere con el planteo teórico 


\section{¿Destrabando una sostenibilidad estéril? Algunas consideraciones en el proceso de selección de indicadores para un diagnóstico territorial}

a priori a lo que se ha llamado frame work o metodología en el proceso de orden y selección de indicadores (GuTIÉRREz-ANTINOPAI, 2017). Por su parte, si la definición de sostenibilidad contempla el resguardo de las generaciones futuras para que satisfagan sus necesidades y, en adición, la incorporación de límites (lo que comúnmente se entiende como la finitud de recursos naturales en el planeta), podría ser un lente interesante en el cómo se percibe, principalmente, porque fue consensuado en sucesivas conferencias y adoptado por gobiernos para la implementación de nuevas políticas y prácticas. Ahora bien, al existir una sobreutilización por parte de variopintas entidades, el término debería examinar dimensiones o variables que delineen cómo construir y seleccionar un sistema de indicadores para que el lobo no termine de vestirse con piel de oveja (de LANDER, 2011).

En este contexto es cuando la sostenibilidad necesita innegablemente un lente de aproximación. De acuerdo con GaLlopín (2003), el lente de sostenibilidad muy débil cumple un rol dominante en la sustitución entre recursos naturales (capital natural) y bienes (capital económico). En tanto, la sostenibilidad débil, si bien permite el resguardo de capital natural crítico, se encuentra supeditada a mecanismos de mercado y crecimiento económico para la protección de resguardo de ecosistemas. De ello se puede concluir que estas visiones encajan con la visión neoliberal tal cual lo indica LANDER (2011) respecto del documento Economía Verde (ONU, 2011). El lente neoliberal, al priorizar lo monetario — sin olvidar la complejidad matemática que existe detrás de la teoría y modelos predictivos-, implica adaptarse y regularse, seguramente, a una valorización crematística del bien o servicio en cuestión. Pérdidas de biodiversidad, estado de océanos, acidificación de suelos tendrían que ajustarse según su potencial para producir o lisa y llanamente para satisfacer las expectativas de un agente consumidor.

En contraste, los fundamentos de la sostenibilidad nacen de una respuesta institucional que intenta acoplar miradas integrales de desarrollo y, ciertamente, en cuya génesis (dígase informes World Conservation Strategy, Brundtland y A21) reside una crítica directa al consumo. Justamente, uno de los lentes que se logró desarrollar sobre la base de la sostenibilidad consistió en velar por el monitoreo de variables asociadas al consumo y desarrollo humanos generando una línea base que fluctúe entre "infraconsumo" y "sobreconsumo" (Spangenberg, 1995; Wautiez y Llavero (2002), Larraín, 2002). Todo ello, dentro del marco de la sostenibilidad fuerte o aquella sostenibilidad que entiende que el intercambio entre capital natural y manufacturado no es sustitutivo (GALLOPín, 2003; BARTON, 2006). 
6. Refiérase a declaraciones Río 92, Estambul 96,

Johannesburgo 2002, Río 2012, Nueva York 2015, Quito 2016.
SALAZAR (2018) indica que efectivamente existen dos tipos de "sostenibilidades" y que una de ellas fue desplazada paulatinamente por consensos liderados por la ONU. La otra, en cambio, si se analizan algunas secciones de informes y declaraciones de diferentes cumbres, cuenta con que existe cierta resistencia que aún puede otorgarnos atisbos de una sostenibilidad fuerte, desprendiéndose, por ejemplo, de guiar hacia la sostenibilidad por medio de sistemas de indicadores que develen niveles de pobreza material y niveles de impacto a la matriz biótica o de recursos naturales mediante apelativos como "mitigación de gases invernadero", "cambios en las modalidades de consumo", "consumo responsable” o "reducción de consumo" (Gutiérrez-Antinopai y Escalona, 2018). Así, en la Cumbre de Desarrollo Sostenible realizada en Nueva York el año 2015 se contempla respecto de la operacionalización de indicadores: "De aquí a 2030, aprovechar las iniciativas existentes para elaborar indicadores que permitan medir los progresos en materia de desarrollo sostenible" (ONU, 2015: 31). Del espectro bien conocido como pobreza, los ámbitos que se incluyen en su medición encuentran un horizonte relacionado con el concepto de desarrollo humano promovido por las Naciones Unidas: "Apoyaremos esfuerzos que definan y refuercen sistemas de monitoreo inclusivos y transparente para reducir la proporción de personas que viven en tugurios y asentamientos informales" (ONU, 2017: 19). Es posible ver este común denominador en otros documentos ${ }^{6}$ : crear aparejamiento de oportunidades mejorando situaciones de educación, salud, vivienda, transporte, y otras, en países en vías de desarrollo. En tercer lugar, de acuerdo con la reducción de niveles de consumo: "Reconocemos que las ciudades y asentamientos humanos enfrentan amenazas sin precedentes que provienen de un consumo insostenible y patrones de producción, pérdida de biodiversidad, presión a los ecosistemas, contaminación...” (ONU, 2017: 18).

La Agenda 21, por su parte, fue pionera en dedicar un capítulo completo llamado Cambio de las modalidades de consumo. En ella se enfatizan prácticas como reutilización, reducción y disminución energética, con el fin de ser eficaces en la creación de nuevos sistemas o en la transición de comportamientos netamente de consumo a unos más sostenibles. La misma agenda va incluso más allá y se cuestiona conceptos de riqueza y prosperidad, denunciando

Se deberían formular nuevos conceptos de la riqueza y la prosperidad en los cuales haya margen para alcanzar niveles de vida más altos, utilizando para ello el cambio de los estilos de vida hacia estilos que dependan menos de los recursos finitos de la Tierra... (ONU, 1992: 6). 
Espacio legítimo ambiental: una sine qua non para la generación de indicadores

En esta línea, retomando a SARA LARRAín (2002) y WAUTIEZ y LLAVERo (2002) y sobre todo a JOACHIM SPANGENBERG ${ }^{7}$ (1995) con el concepto “espacio legítimo ambiental”, nos ayudan a considerar que los indicadores debiesen traducirse en la no privación de consumos básicos y al mismo tiempo velar por el control del sobreconsumo y la equidad social (véase figura 1).
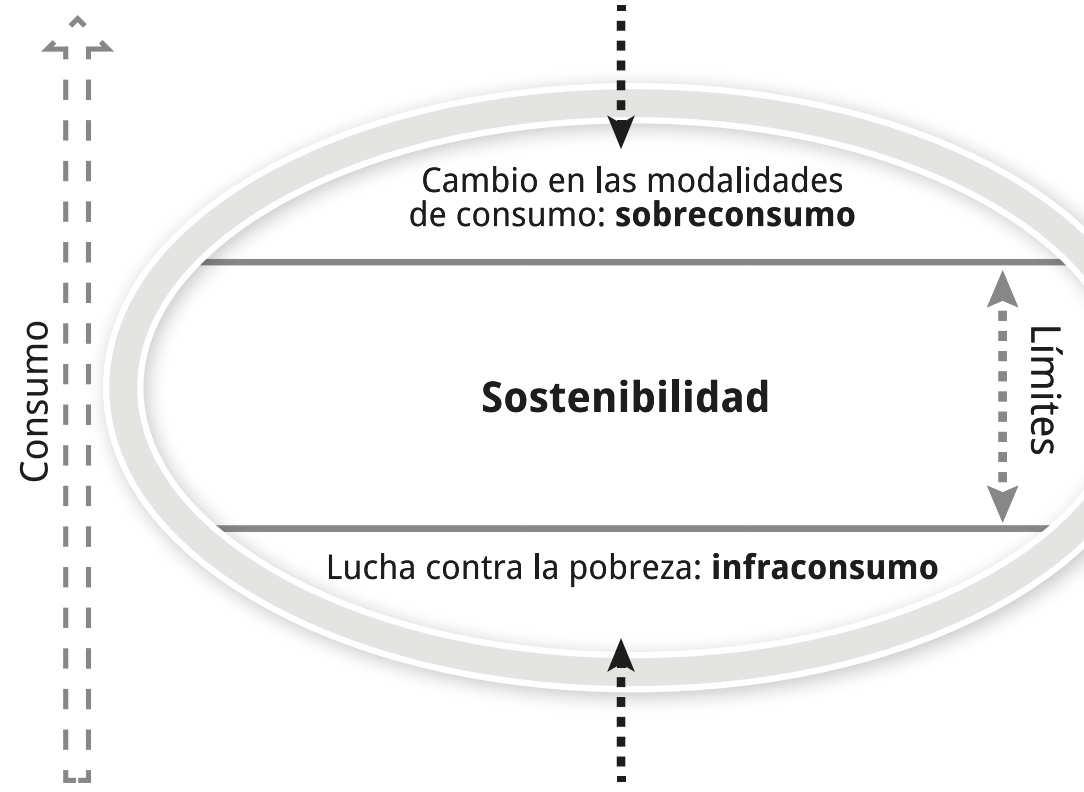

Cambio en las modalidades de consumo: sobreconsumo

Lucha contra la pobreza: infraconsumo

Esto, no obstante, podría crear un escenario de tensión, donde por una parte se difunde incentivar consumo para erradicar problemas básicos de materialidad y, por otro lado, se exhorta a disminuirlo bajo ciertos calificativos que distan del "erradicar" o "poner fin", tal como los objetivos relativos a la superación de la pobreza han estado trazados. En este
7. Su trabajo es retomado por LARRAÍN (2002) y WAUTIEZ Y LLAVERO (2002); tiene por objetivo la redistribución de recursos materiales (superación de la pobreza) respetando los límites bióticos y abióticos del planeta. Cabe destacar que de acuerdo con WAUTIEZ Y LLAVERO (2002) el autor que trabajó inicialmente este concepto fue Hans Opschoor en 1987.

Figura 1. Espacio Legítimo Ambiental. Fuente: SPANGEBERG en GUTIÉRREZ-ANTINOPAI Y ESCALONA (2018) 
8. Algunas de ellas son: Bogotá cómo vamos 2003

(FERNÁNDEZ, 2003), BOGOTÁ CÓMO VAMOS (2015), ISO (2014), BID (2014) y su metodología de indicadores en ciudades intermedias que se han aplicado en varias ciudades de Latinoamérica y el Caribe incluyendo a Valdivia de Chile en el año 2014.

9. Dígase RODRíGUEZ, BARTON, LEón, Solís, CAMPERo y BAEZA (2014); MUÑIZ, RoJAS, BUSULDU, GaRCía, FILIPE Y QUiNTANA (2016), RODRÍGUEZ (2017). contexto, la definición de límites de sostenibilidad en el objetivo 12, "Garantizar modalidades de consumo y producción sostenibles”, presenta un desafío insoslayable y paradójico que enarbola indicadores de emisiones de $\mathrm{CO} 2 \mathrm{y}$, al mismo tiempo, indicadores monetarios como el PIB. Efectivamente, en diferentes experiencias ${ }^{8}$ se observaron indicadores de contaminación definidos por PIB sobre unidades $\mathrm{CO} 2$ generado, que aparentemente cumplen con lo básico para entrar en un sistema de indicadores. Sin embargo, si decimos que el PIB en un período $\left(\delta_{1}\right)$ alcanza las 1000 unidades y el CO2 las 100 unidades, la eficiencia sería de 10; en cambio, si en el siguiente período $\left(\delta_{2}\right)$ el PIB alcanza 3000 unidades y el CO2 las 250 unidades, la eficiencia sería de 12. Este patrón se entendería como algo positivo, más si se analiza el crecimiento de CO2 de 100 a 250 unidades; el crecimiento es significativo, lo cual, si no se indican límites, sería un planteo contraproducente.

Algunos autores para acercarse a esta problemática optan por indicadores de flujo de materiales o huella ecológica ${ }^{9}$. En general, indicadores agregados de escala regional, nacional o mayor que tienden a aglomerar distintas variables para cuantificar y comparar según umbrales máximos permitidos y/o territorios. Realizado bajo Wackernagel y Rees en 1996 permite visibilizar patrones de consumo que han ido en aumento a medida que el PIB aumenta, lo que evidencia la correlación PIB-emisiones. La huella ecológica, quizás siendo el indicador sinóptico o compuesto de mayor renombre, permite mediante la normalización de indicadores llegar a una unidad de fácil asimilación que normalmente es hectáreas per cápita por cada tonelada de CO2 consumida (Rodríguez ET ÁL., 2014). Comprende además una diferenciación entre hectáreas globales o hectáreas locales según la productividad promedio a nivel mundial o local. Verbigracia, New Economic Foundation (2016) considerando hectárea global per cápita estipula un límite de 1,7 hectáreas globales per cápita, que es lo que es capaz de resistir el planeta (biocapacidad) sobre la base de la producción mundial. Por su parte, el Análisis de Flujo de Materiales, metodológicamente introducido por Peter Baccini y Paul Brunner en 1991 (Díaz y Silva , 2015), se utilizó en reiteradas ocasiones como herramienta complementaria a la obtención de la huella ecológica, particularmente, por su modelo de cuantificación de entradas y salidas (flujos output e input) en un asentamiento humano; dicho de otra manera,

La identificación de cada uno de estos flujos exige valorar el comportamiento de las variables que están presentes dentro del sistema elegido y asociadas tanto a la producción y consumo, como a las importaciones y exportaciones de una ciudad o de una región. (RodRíGuEZ ET ÁL., 2014: 42) 


\section{¿Destrabando una sostenibilidad estéril? Algunas consideraciones en el proceso de selección de indicadores para un diagnóstico territorial}

Otra posibilidad es la Apropiación Humana de Producción Primaria Neta (AHPPN o en sus siglas en inglés HANPP), P. Vitousek, P. Ehrlich, A. Ehrlich y A. Matson en 1986 (MARTínEZ-AliER, 1999). Es un indicador que capta lo que las actividades humanas se apropian del residuo de energía derivada de especies autótrofas que utilizan la energía solar como principal motor en sus ciclos de vida. Al provenir de una línea fuera de un orden urbano y más ligado a los ciclos ecológicos, no se considera como un indicador habitual en el desarrollo de experiencias de sistema de indicadores. Aun así, sería interesante cómo podría ayudar a la discusión del tipo de ciudad que se precisa según las necesidades ecosistémicas locales.

Los dos primeros indicadores previamente nombrados transitan en un espectro macroeconómico, por lo que su aplicación suele ser a escalas regionales como mínimo o conurbadas. En tanto, las escalas comunales, municipales y locales habitualmente no utilizan este tipo de regresiones, sino indicadores simples de consumo eléctrico, agua, residuos u otro similar para captar el sobreconsumo (Giampietro, Mayumi y Sorman, 2010). De hecho, estos indicadores, lejos de ser vagas aproximaciones al ser de abstracción baja debido a las unidades físicas que ellos tienen, son utilizados por experiencias locales e incluso para comparar a nivel comunal las brechas de consumo entre cada uno de los territorios, lo que facilita comunicacionalmente umbrales en cada uno de los indicadores (GuTIÉRREz-ANTINOPAI, 2017).

Otro indicador fuera del espectro biofísico pero que se ha utilizado en diversas experiencias a nivel regional y nacional es el Index of Sustainable Economic Welfare (ISEW) gestado por Daly y Cobb en 1989. Basado en el progreso genuino, este índice monetarista evidencia que a medida que el PIB aumenta, el bienestar definido por ISEW tiene tope e incluso disminu$\mathrm{ye}^{10}$. Basándose en el consumo personal e índice de desigualdad distributiva, menos gastos de defensa (costos de producción antes de disponerlos para el consumo y gastos que protegen efectos secundarios, por ejemplo, costos de accidentes automovilísticos) y menos gastos de depreciación natural (de contaminación, de agotamiento de recursos no renovables, es decir, agotamiento de recursos no renovables), se aproxima al espacio legítimo ambiental como un indicador que alberga tanto variables de infraconsumo (consumo personal y desigualdad distributiva) y sobreconsumo (depreciación ambiental), lo cual permite contemplarlo holísticamente, empero, con una abstracción alta a nivel comunicacional, dado que es un índice que no tiene unidades físicas.
10. Véase Costanza et Ál. (2004). 
Sean cuales fueren los indicadores que definan el cielo ambiental (límite entre niveles de sostenibilidad y sobreconsumo), ya sean de alta (en unidades físicas no definidas o monetarias) o baja abstracción (unidades físicas definidas como litros de consumo de agua per cápita anuales), el sistema de indicadores que contemple el espacio legítimo ambiental podría ayudar indudablemente a guiar hacia una planificación sostenible que acople diferentes miradas del "cómo hacer" territorio.

\section{¿Desde qué y en qué contexto(s) un sistema de indicadores?}

Ahora bien, tomando en cuenta que el panorama recientemente esbozado ayuda a un entendimiento del territorio que se quiere diagnosticar, hay elementos primordiales en el momento de seleccionar indicadores. Retomando la paradoja del "milagro económico" de Chile que se describió brevemente en la primera sección, veamos lo que pasa con uno de los sistemas de indicadores promovidos últimamente por el Gobierno de Chile.

Esta experiencia, nacida del Consejo Nacional de Desarrollo Urbano (CNDU y PNUD, 2017), entrega 53 indicadores estructurales separados en nueve compromisos: mejor acceso a servicios y equipamientos públicos básicos, mejor acceso a movilidad sostenible y menores tiempos de viaje, mejor calidad del medio ambiente urbano, mayor integración social y calidad de barrios y viviendas, más y mejor planificación de ciudades y regiones, mayor crecimiento económico inclusivo y sostenible para el desarrollo urbano, mayor protección de nuestro patrimonio cultural, más y mejor participación ciudadana en las decisiones de desarrollo urbano y mejor seguimiento público de las metas de desarrollo urbano sostenible y calidad de vida urbana. Dicho documento al rescatar indicadores de movilidad de acuerdo con partición modal (transporte público, bicicleta y caminata), consumo de agua per cápita, contaminación atmosférica, contaminación de ruido son una apuesta favorable para develar patrones de consumo dentro de zonas urbanizadas. Así también, indicadores de pobreza multidimensional, seguridad, hacinamiento, acceso a servicios de salud ayudan a focalizar recursos para sopesar inequidades y trazar oportunidades para quienes gradualmente $-\mathrm{y}$ muchas veces silenciosamente- fueron desplazados del desarrollo.

Pese a esto, las críticas que se pueden desprender tienen relación con potenciar lo meramente urbano en términos de infraestructura y todo aquello relativo a una economía terciarizada y al aumento de las condiciones de estándar de vida de la población. Es decir, hay 


\section{¿Destrabando una sostenibilidad estéril? Algunas consideraciones en el proceso de selección de indicadores para un diagnóstico territorial}

un énfasis que, al hacer una suma y resta de indicadores, la prioridad esté en el manejo o gestión territorial en torno a la inversión y en menor medida en los impactos a niveles socio-ecológicos que derivan del "crecimiento económico" ¿Se podría entonces decir que lo sostenible es de utilidad meramente ornamental? Efectivamente, la sostenibilidad, en este caso, aunque se utiliza en los apelativos de dimensiones no cumple como un elemento diferenciador respecto de la calidad de vida, por lo que soslaya y olvida los consensos acerca de límites y cambios en los patrones de consumo. Ello podría obedecer en primer lugar al lente preponderante por el cual se percibe el desarrollo y, por tanto, el diagnóstico territorial: no se logran percibir aspectos fundamentales de la sostenibilidad, más bien se centra en un aspecto de calidad de vida no intergeneracional, en el cual esta última se enmarca en aspectos mayormente derivados de una planificación que releva aspectos de igualdad material y gestión de la naturaleza y, en segundo orden, de impacto ambiental. Por ejemplo, en la dimensión Mejor calidad del medio ambiente urbano el indicador de $\mathrm{CO} 2$ per cápita al año que es ampliamente recomendado no es considerado ni siquiera en las dos primeras categorías, estructural y complementario, sino como de segundo orden. Así también el indicador de consumo eléctrico per cápita (probablemente anual) que ayuda a percibir los flujos y las demandas de bienes se encuentra como complementario y no estructural. En segundo lugar, se observa un gran énfasis en aspectos de convivencia e igualdad de oportunidades, pero no de pérdida, por ejemplo, de biodiversidad, siendo que este aspecto es de urgencia nacional principalmente en las zonas de mayor densidad demográfica de Chile: Región Metropolitana de Valparaíso y de Concepción. De la misma forma, resulta llamativo que no se haya incluido el indicador número de automóviles particulares per cápita en la dimensión (compromiso número 2) mejor acceso a movilidad sustentable, cuando Chile ha proyectado un crecimiento acelerado en diversas comunas del país, lo que implica una serie de consecuencias negativas en la movilidad sostenible que incluyen aumento en tiempos de espera en el transporte por medio de buses, aumento de accidentes, de congestión, de contaminación, entre otras. En tercer lugar, la elección de indicadores cuenta con una tradición sectorial, al menos en Chile, que difícilmente incluye un trabajo mancomunado entre ministerios o entre expertos. Esto es evidente en los indicadores propuestos por el CNDU que fueron presididos por el Ministerio de Vivienda y Urbanismo limitando la participación de otros ministerios y por ende, otros intereses. Entonces ¿existirá un espacio para la creación de indicadores que incorporen diferentes ministerios o intereses relacionados con conocer el impacto hacia elementos bióticos? La respuesta 
11. Como una iniciativa con apoyo de una asociación gremial asociada al desarrollo inmobiliario, sus intereses no comparten los fundamentos asociados a la sostenibilidad; es más, ni siquiera es mencionada. Sin embargo, sus inconvenientes serían que la utilización

de Calidad de Vida no considera percepciones subjetivas, elemento esencial en la conceptualización de esta (GUTIÉRREZ-ANTINOPAI Y ESCALONA, 2018) y los criterios materiales que comprimen la dimensión ambiental a una asociada netamente con fenómenos de sanidady salud. probablemente sea un categórico ¡no todavía! Y si lo hay, el medio ambiente y la sostenibilidad se deberían ocupar solo de su campo y no de cómo hacer ciudad, más aun cuando estamos apostando por una sostenibilidad fuerte.

Resulta incluso más lamentable que a partir de estos criterios los modelos de ciudad con los cuales instituciones y personas se sienten representadas o atraídas suelen ser aquellos que en los cuales se vive con altos estándares materiales y para los que paradójicamente el consumo escuetamente sabe de límites. Si bien el documento gestado por el CNDU trata de impulsar cambios hacia una garantía al acceso equitativo a los bienes públicos urbanos, no existe un sentido de al menos incluir indicadores que velen efectivamente por el resguardo de las próximas generaciones, y se sitúa a las comunas de mayor poder adquisitivo, permanentemente, como los modelos que seguir: Providencia, Vitacura y Las Condes (véanse por ejemplo el Índice de Calidad de Vida Urbana ICVU ${ }^{11}$, IEUT y CChC, 2016, 2017 y 2018).

Pues bien, si los sistemas de indicadores son una clara herramienta de políticas públicas, construidos y emplazados en un ambiente sociopolítico cambiante y dinámico, la sostenibilidad difícilmente tendrá una apuesta fija que logre contraponerse a las tendencias dominantes de indicadores de corte neoliberal. Pasa con el ejemplo recientemente mencionado y, por tanto, la pregunta que puede ayudarnos a contextualizar es de orden político: ¿desde qué y en qué contexto los indicadores? Si vemos el problema de que los sistemas de indicadores no incluyen o no se enraízan en la conceptualización de sostenibilidad fuerte, primero podemos indicar la debilidad de gobernanza (BARTON, 2008). La sostenibilidad necesitaría del poder de ella, advirtiendo una planificación como un espacio de límites ambientales no solo para estas generaciones sino para las próximas, cuestionando la hegemonía política-económica y despertando una sostenibilidad aletargada que penetre claramente como "una opción radical... con el pensamiento urbano contemporáneo dominante" (BARTON, 2008: 9). Consiguientemente, la elaboración de sistemas de indicadores debería responder a intereses íntegros que velen por el territorio más allá de lo sectorial o de intereses particulares, que exhiban los diagnósticos no como una selección de indicadores en la cual apenas converge una dialéctica entre dimensiones ambientales, sociales y económicas. En otras palabras, la gestación de sistemas de indicadores debería al menos concebirse como una zona de reflexión donde los indicadores sean la representación explícita de alguna ideología y, obviamente, de cómo se quiere hacer territorio. 


\section{¿Destrabando una sostenibilidad estéril? Algunas consideraciones en el proceso de selección de indicadores para un diagnóstico territorial}

Simultáneamente, si se entiende la gobernanza como aquel gran estadio en el cual se plasman relaciones de poder, al ser la sostenibilidad una visión que permite darnos cuenta de las contradicciones desarrollistas hegemónicas puede contribuir a desestructurar o al menos tensionar intereses que se reflejarían en los indicadores. Después de todo, los indicadores se plasman en un estadio de permanente apropiación, y es posible para cualquier grupo hacer un uso correcto del término. De hecho, iniciativas ciudadanas han generado una movilización de los hacedores de decisión, tal como ha pasado con Seattle (Sustainable Seattle, 2004), que ha sido referente a nivel mundial como iniciativa ciudadana, o Sao Paulo 2012 (Rede Nossa Sao Paulo, 2011), que a partir de una red de diferentes comunidades seleccionó indicadores y metas 2013-2016. Ciertamente, estas organizaciones y comunidades son una contraparte que ayudaría a delinear intereses propios según cada territorio. Además, ayudarían a visibilizar fuentes secundarias e información faltante para rescatar niveles de sobreconsumos, estados bióticos, de biodiversidad urbana no solo a niveles regionales, sino locales; permitirían definir límites y umbrales del "cielo medio ambiental" para proponer metas concretas, tal como ocurre con la pobreza y otros indicadores asociados a inequidades distributivas, y finalmente, visibilizarían a los hacedores de decisión para contribuir con cambios normativos desde lo local.

Por último, resulta importante aclarar que los sistemas de indicadores son una herramienta de planificación que permite informarnos y tomar decisiones. Ahora bien, ¿en qué medida tomar decisiones? o ¿en cuánto influirían los indicadores para tomar decisiones si ellos contemplan el espacio legítimo ambiental como marco metodológico? son algunas de las preguntas que dan cuenta de que los sistemas de indicadores no revertirán por sí mismos el deterioro ambiental y tampoco movilizarán a la sostenibilidad, sino solo podrán acercarnos o aterrizar lo que se entiende por sostenibilidad. Bien se sabe que los impactos del consumo y las dinámicas territoriales son muchas veces silenciosos y, peor aún, juegan frecuentemente con ilusiones individuales: lo que puede ser visto como un mecanismo que facilite una solución termina siendo una práctica llena de espejismos. Por esta razón, el llamado no solo conviene aspectos (post)estructurales, sino personales que resistan al síndrome del consumo. Tal cómo explica Bauman (2007: 185): “disponer del conocimiento, habilidad y determinación necesarios para hacer uso de la capacidad de elección”. 


\section{Bibliografía}

BANCO INTERAMERICANO DE DESARROLLO (2014). Indicadores de la Iniciativa Ciudades Emergentes y Sostenibles. Guía Metodológica.

BARTON, J. (2008). "El poder de la gobernanza: el eslabón "perdido” de la sustentabilidad urbana”. En G. Yañez, A. Orellana, O. Figueroa, \& F. Arenas (Edit.), Ciudad, Poder, Gobernanza (pp. 413-430). Santiago, Chile: Instituto de Estudios Urbanos y Territoriales, Pontificia Universidad Católica de Chile.

BARTON, J. (2006). "Sustentabilidad Urbana como Planificación Estratégica”. EURE, XXXII (96), 27-45. Recuperado el 2 de marzo de 2015.

BAUMAN, Z. (2007) Vida de consumo. México: Fondo de Cultura Económica.

BOGOTÁ CóMO VAMOS (2015). “Indicadores Bogotá Cómo Vamos 2015”. Obtenido de http:// www.bogotacomovamos.org/documentos/informe-de-calidad-de-vida-2015/.

BOISIER, S. (1997) El Vuelo de una Cometa. Una Metáfora para una Teoría del Desarrollo Territorial. Instituto Latinoamericano y del Caribe de Planificación Económica y Social.

BOSSEL, H. (1999) Indicators for Sustainable Development: Theory, Method, Applications. International Institute for Sustainable Development, Winnepeg, Canada. Recuperado el 10 de marzo de 2015, de https://www.iisd.org/pdf/balatonreport.pdf.

CLUB DE ROMA (1971). The Founex Report on Development and Environment. Obtenido de http://www.stakeholderforum.org/fileadmin/files/Earth\%20Summit\%202012new/Publications\%20and\%20Reports/founex\%20report\%201972.pdf.

CONSEJO NACIONAL DE DESARROLLO URBANO Y PROGRAMA DE LAS NACIONES UNIDAS PARA EL DESARROLLO. (2017). "Sistemas de Indicadores y Estándares de Calidad de Vida y Desarrollo Urbano”. Santiago: Documento realizado por DESE-FADEU de PUC para CNDU.

COSTANZA, R., ERICKSON, J., FLIGGER, K., ADAMS , A., ADAMS , C., ALTSCHULER, B., Y OTROS (2004). "Estimates of the Genuine Progress Indicator (GPI) for Vermont, Chittenden County and Burlington, from 1950 to 2000”. Ecological Economics (51), 139-155.

DAHER, A. (2015). "Cluster minero sin cluster social: Antofagasta-Chile”. Revista de Urbanismo (33), 25-35.

DALY, H., \& COBB, J. (1997) Para el bien común (primera reimpresión español ed.). Distrito Federal, México: Fondo de Cultura Económica.

FERNÁNDEZ, M. F. (2003). "Evaluación de los Cambios de Calidad de Vida en Bogotá”, desde la Sociedad Civil Bogotá Cómo Vamos.

GALLOPÍN, G. (2003) Sostenibilidad y desarrollo sostenible: un enfoque sistémico. Santiago: Comisión Económica para América Latina y el Caribe. 
GEORGESCU-ROEGEN, N. (1996) La Ley de la Entropía y el Proceso Económico. (L. Gutiérrez Andrés, \& M. V. López Paños, Trads.) España: Fundación Argentaria, Visor Distribuciones.

GIAMPIETRO, M., MAYUMI, K., \& SORMAN, A. (2010). "Assessing the quality of alternative energy sources: Energy Return On the Investment (EROI), the Metabolic Pattern of Societies and Energy Statistics". Working Papers on Environmental Sciences. Universitat Autònoma de Barcelona. GUIMARAES, R. (1998) Aterrizando una Cometa: Indicadores Territoriales de Sustentabilidad. Santiago, Chile: Instituto Latinoamérica y del Caribe de Planificación Económica y Social.

GUIMARAES, R. (1994). "El Desarrollo Sustentable: ¿Propuesta alternativa o retórica neoliberal?”. EURE, XXI (61), 41-56.

GUTIÉRREZ-ANTINOPAI, F. (2017). "De indicadores ortodoxos a indicadores de sustentabilidad urbana. Una propuesta de sistemas de indicadores para las comunas urbanas de Chile". Santiago, Chile: Tesis para el grado de MSc en Asentamientos humanos y medio ambiente de la Pontificia Universidad Católica de Chile. Profesor Guía: Jonathan Barton.

GUTIÉRREZ-ANTINOPAI, F., \& ESCALONA, D. (2018). "Indicadores para la sustentabilidad: fundamentos Pre-Agenda 21 para un diagnóstico territorial”. Revista de la Academia, XXVI (primavera), 114-139.

INSTITUTO DE ESTUDIOS URBANOS Y TERRITORIALES Y CÁMARA CHILENA DE LA CONSTRUCCIÓN. (2018). “Índice de Calidad de Vida Urbana, ICVU”. Obtenido de http://www.cchc. cl/comunicaciones/noticias/indice-de-calidad-de-vida-urbana-icvu-2018.

INSTITUTO DE ESTUDIOS URBANOS Y TERRITORIALES Y CÁMARA CHILENA DE LA CONSTRUCCIÓN. (2016). "ICVU 2016 Indicador de Calidad de Vida de Ciudades Chilenas". Santiago, Chile. Recuperado el 15 de diciembre de 2016, de http://www.cchc.cl/uploads/comunicacion/archivos/Presentacio\%CC\%81n_ICVU_2016.pdf.

INSTITUTO DE ESTUDIOS URBANOS Y TERRITORIALES Y CÁMARA CHILENA DE LA CONSTRUCCIÓN. (2017). "Índice de Calidad de Vida, ICVU". Obtenido de http://estudiosurbanos. uc.cl/images/investigaciones/Arturo_Orellana/ICVU_2018/20180508_ICVU_2018_-_Version_ Definitiva.pdf.

INTERNATIONAL ORGANIZATION FOR STANDARDIZATION (2014). "ISO 37120. Sustainable development of communities. Indicators for city services and quality of life".

INTERNATIONAL UNION FOR CONSERVATION OF NATURE, UNITED NATIONS ENVIRONMENT PROGRAMME \& WORLD WILDLIFE FUND (1980). World Conservation Strategy. Living resources conservation for sustainable development.

LANDER, E. (2011). "La Economía Verde: el lobo se viste con piel de cordero". Obtenido de El Transnational Institute: https://www.tni.org/es/colecci\%C3\%B3n/academicos-y-academicas-asociados. 
LARRAÍ́N, S. (2002). "Línea de Dignidad como Indicador de Sustentabilidad Socioambiental". En M. P. Aedo, \& S. Larraín (Edits.), Línea de Dignidad. Desafíos sociales para la sustentabilidad (págs. 89-108). Santiago.

LARRAÑAGA, O., \& RODRÍGUEZ, M. (2014) Desigualdad de Ingresos y Pobreza en Chile 1990 a 2013. Programa de las Naciones Unidas para el Desarrollo, Santiago.

MARTÍNEZ-ALIER, J. (1999) Introducción a la Economía Ecológica. Barcelona: RUBES.

MEADOWS, D. (1998) Indicators and Informations for Sustainable Development. The Sustainable Institute. Recuperado el 28 de Febrero de 2015, de http://www.iisd.org/pdf/s_ind_2.pdf.

MUÑIZ, I., ROJAS, C., BUSULDU, C., GARCÍA, A., FILIPE, M., \& QUINTANA, M. (2016). "Forma urbana y Huella Ecológica en el Área Metropolitana de Concepción (Chile)”. EURE, XLII (127), 209-230.

NAESS, A. (2007). "Los movimientos de la ecología superficial y la ecología profunda: un resumen”. Revista Ambiente y Desarrollo, I (23), 98-101.

NAREDO, J. M. (2007) Raíces económicas del deterioro ecológico y social (Primera reimpresión ed.). España: Siglo XXI.

NEW ECONOMICS FOUNDATION (2016). “The Happy Planet Index 2016”. A global index of sustainable wellbeing.

NOLL, H.-H. (2016). "Progress: Concept and Measurement”. En F. Maggino (Ed.), A Life Devoted to Quality of Life (pp. 93-104).

NOLL, H.-H. (2002). "Social Indicators and Quality of Life Research: background, achievements and current trends”. En N. Genov (Ed.), Advances in Sociological Knowledge over Half a Century.

ORGANIZACIÓN DE LAS NACIONES UNIDAS (1972). "Declaration of the United Nations Conference on the Human Environment”. Estocolmo. Obtenido de http://www.un-documents. net/unchedec.htm.

ORGANIZACIÓN DE LAS NACIONES UNIDAS (1992). “Agenda 21”. Río de Janeiro, Brasil. Obtenido de http://www.un.org/esa/sustdev/documents/agenda21/spanish/a21_summary_spanish.pdf.

ORGANIZACIÓN DE LAS NACIONES UNIDAS (2015). "Transforming Our World: The 2030 Agenda for Sustainable Development”. Asamblea General Naciones Unidas. Obtenido de http://unctad.org/meetings/es/SessionalDocuments/ares70d1_es.pdf.

ORGANIZACIÓN DE LAS NACIONES UNIDAS (2017). "New Urban Agenda”. Quito, Ecuador. Obtenido de http://habitat3.org/wp-content/uploads/NUA-English.pdf.

RASSE, A. (2016) Segregación Residencial Socioeconómica y Desigualdad en las Ciudades Chilenas. Programa de las Naciones Unidas para el Desarrollo, Santiago. 


\section{¿Destrabando una sostenibilidad estéril? Algunas consideraciones en el proceso de selección de indicadores para un diagnóstico territorial}

REDE NOSSA SAO PAULO, PROGRAMA CIDADES SUSTENTÁVEIS (2012). "Indicadores e Referências de Metas para Sao Paulo 2013-2016”. Obtenido de https://www.nossasaopaulo. org.br/portal/files/publicacao-indicadores-e-referencias-de-metas-2013-2016.pdf.

RODRÍGUEZ SALVO, F. (2017). "Hacia un Gran Temuco Bajo en Carbono: Una propuesta metodológica para un territorio bajo en carbono". Tesis para el grado de MSc en Asentamientos humanos y medio ambiente de la Pontificia Universidad Católica de Chile. Profesor guía: Jonathan Barton.

RODRÍGUEZ, C., BARTON, J., LEÓN, S., SOLÍS, O., CAMPERO, C., \& BAEZA, S. (2014). ¿Cuán Sustentable es la Región de Antofagasta? Indicadores y tendencias para un desarrollo regional. Disponible en https:/www.politicaspublicasdelnorte.cl/web/wp-content/uploads/2018/08/ Cu\%C3\%A1n-Sustentable...pdf.

SALAZAR, G. (2018). "The Incongruities of Sustainability: An examination of the UN Earth Summit Declarations 1972-2012”. En P. Spinozzi, \& M. Mazzanti (Edits.), Routing Sustainable Development Towards a Culture of Wellbeing (pp. 46-62).

SCHUSCHNY, A., \& SOTO, H. (2009) Guía Metodológica Diseño de Indicadores Compuestos de Desarrollo Sostenible. Santiago, Chile: Comisión Económica para América Latina y el Caribe.

SPANGENBERG, J. (1995). “Integración de criterios en el concepto de sustentabilidad”. Espacios (7).

SUSTAINABLE SEATTLE. (2004) Indicators of Sustainable Community 1998. Obtenido de http://www.communityindicators.net/system/publication_pdfs/72/original/S21998IndicatorsReport.pdf?1385414709.

TURNER, K. (1993). "Sustainable Environmental Economics and Management”. (K. Turner, Ed.) London: Belhaven Press.

WARD, B., \& DUBOS, R. (1972) Una Sola Tierra: El Cuidado y Conservación de un Pequeño Planeta. D. F. México: Fondo de Cultura Económica.

WAUTIEZ, F., \& LLAVERO, Á. (2002). "La Equidad Socio-ambiental en Chile: Una tarea pendiente”. En M. P. Aedo, \& S. Larraín (Edits.), Línea de Dignidad. Desafíos sociales para la Sustentabilidad (pp. 11-55).

WORLD COMMISSION ON ENVIROMENT AND DEVELOPMENT (1987). "Our Common Future”. Organización de las Naciones Unidas. Obtenido de http://www.un-documents.net/ our-common-future.pdf.

YALE UNIVERSITY, COLUMBIA UNIVERSITY \&. (2018). "Environmental Performance Index: Chile”. Obtenido de https://epi.envirocenter.yale.edu/epi-country-report/CHL. 
